\title{
Fast evaluation of a linear number of local exchange matrices
}

\author{
WanZhen Liang ${ }^{a}$, Yihan Shao ${ }^{\mathrm{b}}$, Christian Ochsenfeld ${ }^{\mathrm{c}}$, \\ Alexis T. Bell ${ }^{\text {a }}$, Martin Head-Gordon ${ }^{b, *}$ \\ a Department of Chemical Engineering, University of California, Berkeley, CA 94720-1460, USA \\ ${ }^{\mathrm{b}}$ Department of Chemistry, University of California, Berkeley, CA 94720-1460, USA \\ c Institut für Physikalische Chemie, Universität Mainz, D-55099 Mainz, Germany
}

Received 18 December 2001; in final form 18 March 2002

\begin{abstract}
A fast method is described for evaluating multiple exchange matrices in a Gaussian atomic orbital basis. For insulators, it is asymptotically linear scaling, and is a generalization of the linear scaling exchange (LinK) method, which was formulated for a single exchange matrix [J. Chem. Phys. 109 (1998) 1663]. It is employed to evaluate exchange-type contractions of all derivative density matrices with two-electron integrals for a series of linear alkanes, linear polyacenes, and water clusters using STO-3G, 3-21G, and 6-31G* basis sets. Significant computational savings are obtained for molecules with as few as 10 non-hydrogen atoms. (c) 2002 Published by Elsevier Science B.V.
\end{abstract}

\section{Introduction}

In self-consistent field (SCF) methods, there are three computationally intensive contributions to the effective Hamiltonian. First is the purely local exchange-correlation potential which can be evaluated by linear scaling numerical quadrature [1-3]. The second and third contributions arise from four-center, two-electron repulsion integrals (ERIs), and are the direct Coulomb potential and the so-called exact-exchange potential. Linear scaling methods are available for both of these latter terms in the SCF procedure. The Coulomb terms are efficiently evaluated using variants of the fast multipole method (FMM) [4-10].

\footnotetext{
${ }^{*}$ Corresponding author. Fax: +1-510-643-1255. don).
}

Our focus in this Letter is on the exchange problem, for which several $\mathrm{O}(N)$ methods which exploit the locality of the density matrix have been developed [11-17]. Some of these methods are based on truncating density matrices and exchange matrices with distance dependent cutoffs to achieve linear scaling of computational time with system size $[11,12,18]$. The first method that did not assume decay properties was described in [13]. Then, in $[16,17]$, a linear scaling method (LinK) to form the exact Hartree-Fock exchange matrix and exchange gradients was presented, which preserves the highly optimized structure of conventional direct SCF methods [19] with only negligible prescreening overhead and does not impose predefined decay properties. The present development is a generalization of this LinK method.

Almost all of this previous work applies to the case where just a single exchange matrix is 
evaluated from a given set of ERIs. However, there are several important applications of exchange (and Coulomb) matrices, where the number of matrices to be evaluated is proportional to the size of the system. The first and most important is the problem of analytical force constants in SCF theory, which are required to predict vibrational frequencies, characterize stationary points, find reaction paths, etc. Analytical SCF force constants require solution of the coupled perturbed SCF (CPSCF) equations for each independent nuclear displacement [20-23], which in turn requires the exchangetype contraction of all derivative density matrices with the two-electron integrals for the evaluation of derivative exchange matrices $K^{x}$ for all displacements $x$ (the derivatives of the two-electron integrals are not considered in the present Letter). In principle, this must scale one power of system size higher than the evaluation of a single exchange matrix so that for the conventional direct CPSCF algorithm [21], cubic scaling results. For the LinK algorithm [16,17] the scaling is asymptotically independent of system size for the contraction of one perturbed density matrix (due to its locality for insulators), so that an overall linear scaling can result for the contraction with all derivatives. However, the contraction of each density-matrix derivative, one at a time, with the ERIs has clear disadvantages, since one needs to recalculate the integrals for each density. Therefore a simultaneous contraction of all derivative quantities is highly desirable. However, if done in a straightforward way such a simultaneous contraction would lead to conventional cubic scaling. In this Letter we introduce a new algorithm to (asymptotically) achieve linear scaling for the simultaneous contraction of multiple matrices for the formation of $K^{x}$ and similar exchange-type quantities.

This algorithm is based on the fact that for insulators, in the atomic orbital basis, the derivative density matrix for an atomic displacement $x$ has been shown to decay exponentially with basis function separation, and also very rapidly with separation of the functions from the perturbed atom [24]. Thus asymptotically only a constant number of derivative density matrix elements are non-zero for a single matrix. This raises the possibility of achieving linear scaling not only for the
SCF problem itself but also for the solution of the CPSCF equations. To allow for the linear scaling in solving the CPSCF equations a reformulation of the conventional equations is necessary which has been done in a density matrix based fashion in the atomic orbital basis first by Ochsenfeld and HeadGordon [24] and later by Larsen et al. [25]. It clearly requires the ability to evaluate $\mathrm{O}(N)$ local exchange matrices $K^{x}$ with only $\mathrm{O}(N)$ effort, on each CPSCF iteration. The problem of efficient evaluation of Coulomb interactions is also essential, but will not be addressed here: there are some preliminary efforts in this direction already [26,27].

A second area in which multiple local exchange (and Coulomb) matrices arise is in local formulations of coupled cluster (CC) theory. For example, in the perfect pairing [28], imperfect pairing [29], and restricted pairing [30] theories of bondbreaking, on each iteration of the orbital equations, one must evaluate local Coulomb and exchange matrices. The required exchange matrices are $K^{i i}, K^{i^{*} i}$ and $K^{i^{*} i^{*}}$ for each valence electron pair, described by an orbital pair index $\left(i, i^{*}\right)$, where these orbitals $i$ and $i^{*}$ are typically local bonding and antibonding orbitals, respectively. The corresponding local density matrices are

$P_{\mu v}^{i i}=C_{\mu i} C_{v i}$

$P_{\mu v}^{i i^{*}}=C_{\mu i} C_{v i^{*}}$,

$P_{\mu \nu}^{i^{*} i^{*}}=C_{\mu i^{*}} C_{v i^{*}}$.

Here $C$ are the molecular orbital coefficients, and the indexes $i$ and $i^{*}$ run over all pairs of valence electrons. Clearly this problem is fundamentally similar to the CPSCF problem: naive evaluation of these exchange matrices scales as $\mathrm{O}\left(N^{3}\right)$, but since they each have asymptotically only a constant number of elements, linear scaling appears possible.

\section{Algorithm description}

In this section, we present a simple description of our numerical algorithm for forming multiple exchange matrices $K^{x}$, where 
$K_{\lambda \sigma}^{x}=\sum_{\mu \nu} P_{\mu v}^{x} \cdot(\lambda \mu \mid \sigma v)$,

and $P_{\mu v}^{x}$ is a local derivative density matrix, indexed by either Cartesian atomic displacements (for the CPSCF problem) or by electron pair number (for the local CC problem). Our new scheme to evaluate these multiple exchange matrices involves two main steps to reduce computational time. One is to reduce the number of four-center two-electron repulsion integrals
(ERIs) which are evaluated to just a linear number $[11-14,16]$. This first step is closely related to the conventional LinK scheme $[16,17]$ and requires only minor changes as compared to the original scheme. The second step is new and crucial to reduce the number of floating point operations involved in contracting each of the $\mathrm{O}(N)$ integrals with up to $\mathrm{O}(N)$ densities simultaneously to also just a linear number. We shall discuss both steps in turn. The algorithm is summarized in Figs. 1 and 2.

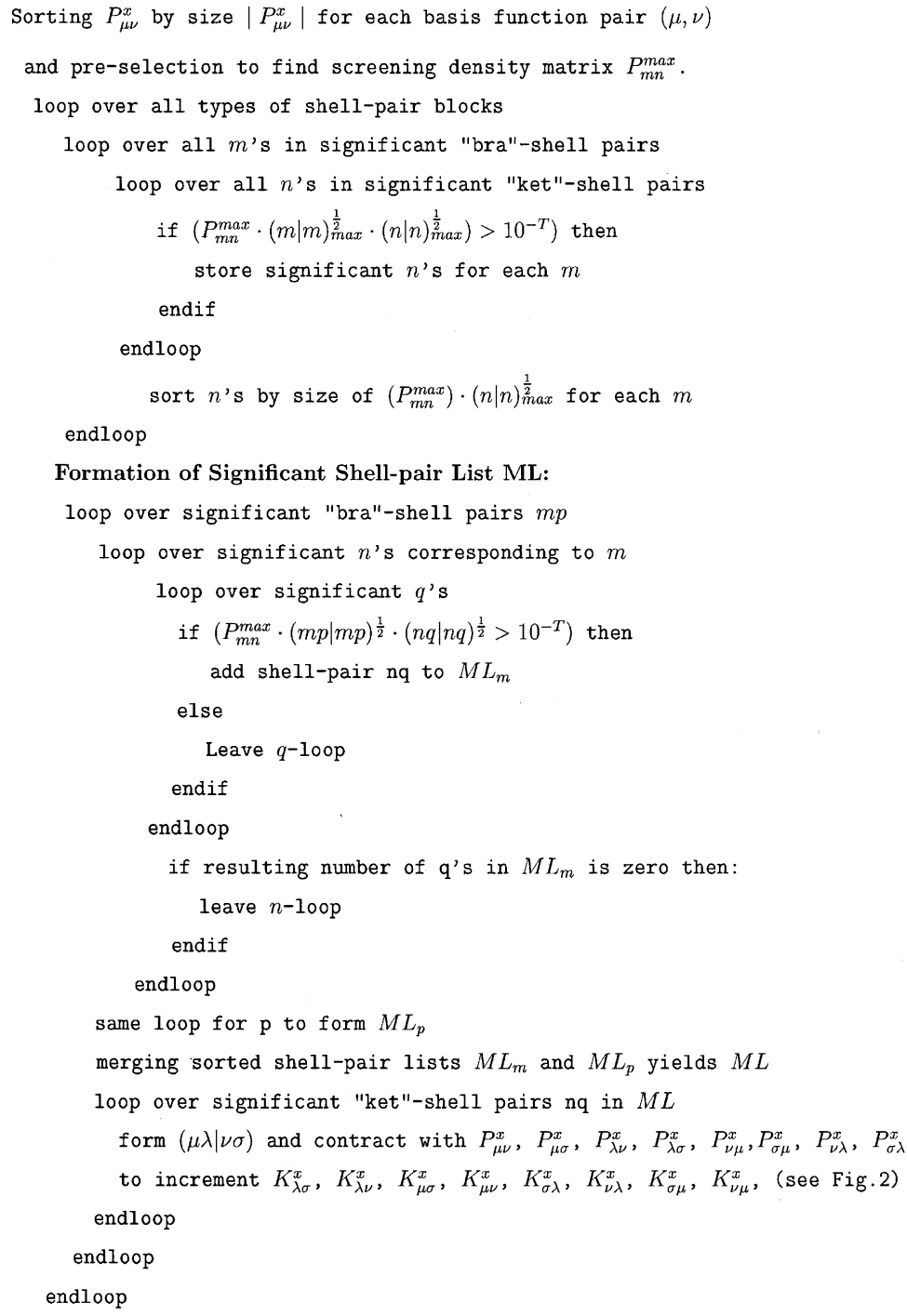

Fig. 1. Overall loop structure for the simultaneous formation of multiple local exchange matrices $K^{x}$. The algorithm is not constrained to symmetric $P^{x}$ for which e.g. $P_{\mu v}^{x}$ would be of course equal to $P_{v \mu}^{x}$. 


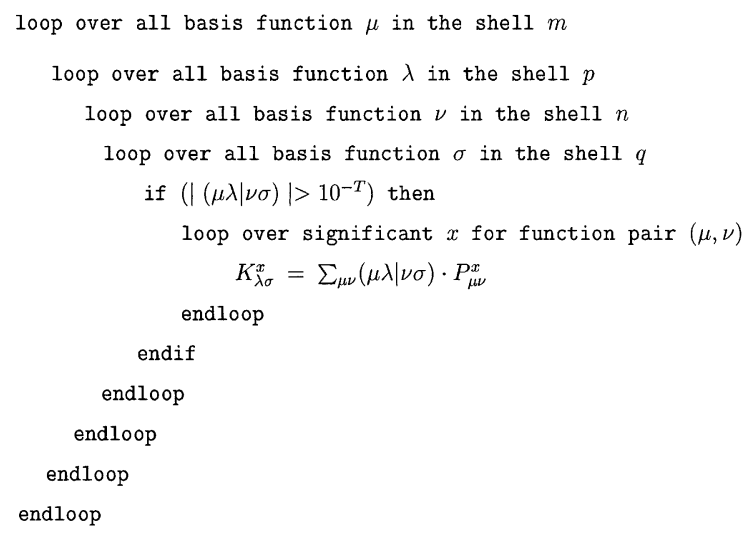

Fig. 2. This loop structure is employed to increment $K_{\lambda \sigma}^{x}$ elements. Here the number of the significant $x$ is chosen according to $\left|P_{\mu v}^{x}\right| \geqslant 10^{-(T-\mathrm{IX})}$ for function pair $(\mu, v)$. Here IX $=$ $\operatorname{INT}\left(-\log _{10}|(\mu \lambda \mid v \sigma)|\right)$.

The very first step is an initial sort, where we order the density matrix derivatives $P_{\mu \nu}^{x}$ for each pair of basis function $\mu$ and $v$ by their magnitudes, $\left|P_{\mu \nu}^{x}\right|$. If the matrices were provided in dense form, this is also the point where insignificant values are discarded. These sorted density matrices will be exploited in the final step of contractions with the two-electron integrals. It will allow only a limited number of density matrix elements (i.e. a limited number of significant values of $x$, which asymptotically becomes independent of molecular size) for each basis function pair index $\mu$ and $v$ to be contracted with the two-electron integrals that have been produced.

Based on the multiple sets of local density matrices, $P_{\mu \nu}^{x}$, we also pre-form a single screening density, $P_{m n}^{\max }$, for each significant shell pair, $(m, n)$, based on

$P_{m n}^{\max }=\max _{x, \mu \in m, v \in n}\left(\left|P_{\mu \nu}^{x}\right|\right)$.

$P_{m n}^{\max }$ is the maximum magnitude of all the density elements involved in the $(m, n)$ shell-pair block, for all $x . P_{m n}^{\max }$ will be employed to find the significant shell-quartets.

We are now ready to discuss how to generalize the original LinK approach [16] to pre-screening the two-electron integrals

$$
\begin{aligned}
(\mu \lambda \mid v \sigma)= & \int \mathrm{d} \mathbf{r} \int \mathrm{d} \mathbf{r}^{\prime} \psi_{\mu}^{*}(\mathbf{r}) \psi_{\lambda}^{*}(\mathbf{r}) \\
& \frac{1}{\left|\mathbf{r}-\mathbf{r}^{\prime}\right|} \psi_{v}\left(\mathbf{r}^{\prime}\right) \psi_{\sigma}\left(\mathbf{r}^{\prime}\right) .
\end{aligned}
$$

An AO basis of dimension $N$ produces $\mathrm{O}\left(N^{4}\right)$ four-center ERIs, of which $\mathrm{O}\left(N^{2}\right)$ are significant in a large system [31,32]. The problem is how to reduce this number to $\mathrm{O}(N)$ by exploiting the locality of the matrix/matrices to be contracted $[11-14,16]$. This first part of the method is analogous to the original LinK scheme, but is necessary to understand the following step of screening in the contraction step of the multiple matrices. We have available the Schwartz inequality [33] to bound the magnitudes of the ERIs:

$$
|(m p \mid n q)| \leqslant(m p \mid m p)^{1 / 2} \cdot(n q \mid n q)^{1 / 2} \text {. }
$$

Here $m, n, p$, and $q$ denote the indices of shells, $\mu$, $v, \lambda$, and $\sigma$ denote the indices of AO orbitals within these shells. Use of shell structure is essential to efficient ERI evaluation [34]. The other information we have available for pre-screening is the connection between the density matrices and the magnitude of exchange contributions via Eq. (4).

The next step lies within the main outer loops over bra and ket shell pairs of a given type (common angular momentum and degrees of contraction). The number of such quartet types is independent of system size. The pre-screening is focused one by one on each different bra shell, $m$, that occurs in the list of bra shell pairs of the current type. For each $m$, a set of distinct ket shells, $n$, are chosen from all ket shells within the current ket shell pairs according to the following criterion:

$\left(P_{m n}^{\max }\right) \cdot(m \mid m)_{\max }^{1 / 2} \cdot(n \mid n)_{\max }^{1 / 2} \geqslant 10^{-T}$.

Here $(m \mid m)_{\max }=\max _{p \in m p}(m p \mid m p)$ and $(n \mid n)_{\max }=$ $\max _{q \in n q}(n q \mid n q)$ are the maximum over all integral estimates of these shell-pair types, that involve shell $m$ and $n$, respectively. These lists will be used later to aid in selecting significant shell quartets. Asymptotically, this will reduce the number of significant $n$ 's for a given $m$ to only a constant value rather than the linear number that would otherwise be selected. 
After all significant $n$ 's for a given $m$ have been identified, they are ordered by the size of their density-weighted integral estimates,

$\left(P_{m n}^{\max }\right) \cdot(n \mid n)_{\max }^{1 / 2}$.

In the subsequent shell quartet selection loops, this density-weighted integral ordering allows immediate loop exits once a certain threshold criterion $10^{-T}$ is reached $[13,16]$, and is crucial to permit efficient linear scaling in the selection procedure.

At this point we enter the production loop over all of the bra shell pairs $(m, p)$ of the current type. For each one, our task is to use the lists constructed above to select (just the constant number of) significant ket shell pairs of the current type. For shell $m$ we loop over the significant $n$, as shown in Fig. 1, yielding a mini-list (ML) by the following screening criterion:

$P_{m n}^{\max } \cdot(m p \mid m p)^{1 / 2} \cdot(n q \mid n q)^{1 / 2} \geqslant 10^{-T}$.

The cost of this selection is only linear because in addition to the significant $n$ ordering, shell pairs have already been pre-selected and pre-ordered by the size of the integral estimate in Q-CHем [35] into which we implemented our new method. Additionally we repeat this process for significant ket shells $q$ associated with bra shell $p$, and merge the two minilists together. This defines the ket shells to be combined with the current bra shell.

For the significant shell-quartets selected in this way, we can proceed to produce and contract the significant two-electron integrals $(\lambda \mu \mid v \sigma)$ with significant density matrix elements $P_{\mu v}^{x}$ to increment matrices $K^{x}$. This contraction is described in the following and is the crucial step of the generalized LinK scheme for simultaneous contraction of multiple matrices. It differs from the original LinK method, since it is potentially an $\mathrm{O}\left(N^{2}\right)$ step, even after ERI evaluation has been reduced to $\mathrm{O}(N)$, given that there are $\mathrm{O}(N)$ separate density and exchange matrices.

All elements $P_{\mu \nu}^{x} \cdot(\mu \lambda \mid v \sigma)$ which are smaller than the threshold $10^{-T}$ are discarded. Here for each basis function pair, $(\mu, v)$, only an asymptotically constant number of $x$ values instead of $\mathrm{O}(N)$ (or to be exact three times the number of atoms in CPSCF or three times the number of doubly occupied molecular orbitals in the local CC methods)
$P_{\mu \nu}^{x}$ will be contracted with each significant twoelectron integral. The loop structure shown in Fig. 2 is employed to perform this contraction. It permits the number of significant $x$ values to be determined based on the magnitude of the computed ERI, and also avoids contraction entirely if the magnitude of the ERI is below threshold.

\section{Results}

In the following we present timings for our new algorithm to explore its performance relative to a conventional method where only the two-electron integrals are thresholded with a threshold of $10^{-11}$. All calculations are for the problem of evaluating the local exchange matrices corresponding to the derivative density matrices obtained when the CPSCF equations are converged (to $10^{-6}$ for Hartree-Fock theory) [20]. Identical integral evaluation methods are used in the conventional and linear scaling algorithms. All other parts of the calculation apart from exchange matrix evaluation are excluded in the timings. The calculations are for three classes of model systems: one-dimensional large-gap linear alkanes, one-dimensional small-gap linear polyacenes, and threedimensional large-gap water clusters. Three different basis sets (STO-3G, 3-21G and 6-31G*) are used to examine basis set effects on the performance of the algorithm. All timings were obtained using a development version of the QCHem program package [35] on a $375 \mathrm{MHz}$ IBM Power-3 workstation (Model 270).

Fig. 3 for the linear alkanes shows computational time versus the number of basis functions for the new algorithm and for the conventional algorithm. This is for the minimal STO-3G basis set and compares two different numerical thresholds, $10^{-7}$ and $10^{-11}$ in the new algorithm. Comparing against the conventional calculations, significant computational savings are observed with the new algorithm. CPU time is reduced by factors of 1.7 and 1.0 for $\mathrm{C}_{6} \mathrm{H}_{14}$, by 12.2 and 3.3 for $\mathrm{C}_{24} \mathrm{H}_{50}$, and by 16.6 and 4.6 for $\mathrm{C}_{30} \mathrm{H}_{62}$, with thresholds of $10^{-7}$ and $10^{-11}$ respectively. The computational time asymptotically scales linearly with molecule size, and this scaling behavior ap- 


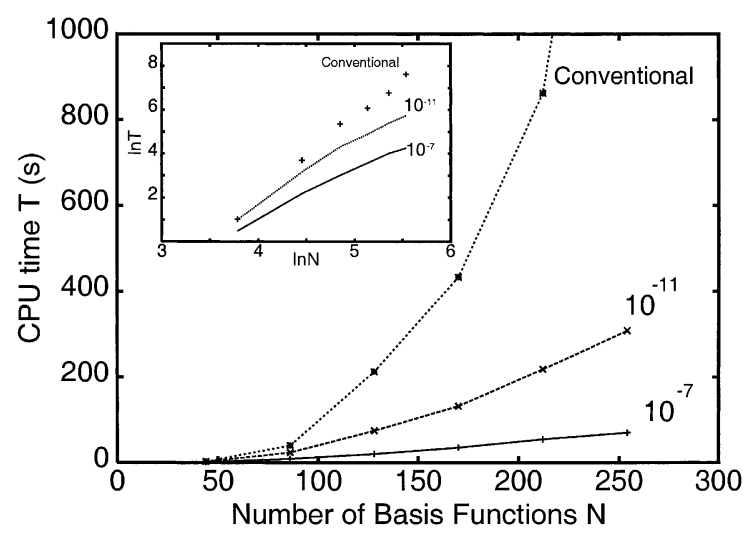

Fig. 3. CPU time $T$ versus the number of basis functions $N$ using the conventional method and the new algorithm for evaluating the multiple exchange matrices for linear alkanes with different thresholds. The solid line: new algorithm with a threshold of $10^{-7}$, the dashed line: new algorithm with a threshold of $10^{-11}$, the dotted line: the conventional calculation with an integral threshold of $10^{-11}$. The inset plots $\ln T$ versus $\ln N$. pears to be reached for a few dozens of non-hydrogen atoms with STO-3G basis set and a threshold of $10^{-7}$ for linear alkanes. At the higher threshold, linear scaling is not yet achieved for the calculations shown in this figure.

To more clearly show the scaling behavior of CPU time $T$ versus the number of basis functions $N$, the inset of Fig. 3 plots $\ln T$ via $\ln N$. Surprisingly, the conventional calculations shows a scaling factor that does not straightforwardly decrease to an asymptotic value of 3 . This appears to reflect cache miss effects in the larger calculation. By contrast, the new method shows a gradually reducing scaling factor $\alpha$ as molecular size increases. This case (large-gap insulator and small basis set) is the most favorable one for the new algorithm. We turn next to some examples that are more challenging (larger basis sets and/or smaller gaps meaning longer-ranged density matrices).

Table 1 displays the computational time versus molecular size when different basis sets are em-

Table 1

CPU time and numerical errors for evaluation of multiple exchange matrices using different basis sets for linear alkanes, water clusters and linear polyacenes with thresholds of $10^{-7}$ and $10^{-11}$

\begin{tabular}{|c|c|c|c|c|c|}
\hline Molecule & Basis set & $\begin{array}{l}\mathrm{CPU}_{10^{-7}} \\
\text { (s) }\end{array}$ & $\begin{array}{l}\mathrm{CPU}_{10^{-11}} \\
\text { (s) }\end{array}$ & $\begin{array}{l}\mathrm{CPU}_{\text {con }} \\
\text { (s) }\end{array}$ & Error $_{10^{-7}}$ \\
\hline \multirow[t]{3}{*}{$\mathrm{C}_{12} \mathrm{H}_{26}$} & STO-3G & 9.12 & 23.23 & 38.00 & $6.94 \times 10^{-6}$ \\
\hline & $3-21 G$ & 52.06 & 163.66 & 308.49 & $4.34 \times 10^{-6}$ \\
\hline & $6-31 G^{*}$ & 174.17 & 580.65 & 1155.81 & $1.33 \times 10^{-5}$ \\
\hline \multirow{3}{*}{$\mathrm{C}_{24} \mathrm{H}_{50}$} & STO-3G & 35.37 & 132.40 & 432.52 & $5.16 \times 10^{-6}$ \\
\hline & $3-21 G$ & 201.16 & 888.21 & 2782.21 & $1.09 \times 10^{-5}$ \\
\hline & $6-31 \mathrm{G}^{*}$ & 637.60 & 3150.16 & 12417.52 & $2.22 \times 10^{-5}$ \\
\hline \multirow{3}{*}{$\left(\mathrm{H}_{2} \mathrm{O}\right)_{10}$} & STO-3G & 3.37 & 12.41 & 19.94 & $4.74 \times 10^{-6}$ \\
\hline & $3-21 G$ & 14.40 & 54.39 & 92.01 & $1.18 \times 10^{-5}$ \\
\hline & $6-31 G^{*}$ & 54.66 & 255.66 & 484.59 & $1.15 \times 10^{-5}$ \\
\hline \multirow{3}{*}{$\left(\mathrm{H}_{2} \mathrm{O}\right)_{20}$} & STO-3G & 18.48 & 104.17 & 315.69 & $5.89 \times 10^{-5}$ \\
\hline & $3-21 G$ & 98.13 & 474.17 & 1429.38 & $2.75 \times 10^{-5}$ \\
\hline & 6- $31 \mathrm{G}^{*}$ & 347.64 & 2072.74 & 7617.82 & $3.92 \times 10^{-5}$ \\
\hline \multirow{2}{*}{$\left(\mathrm{H}_{2} \mathrm{O}\right)_{30}$} & STO-3G & 49.51 & 290.77 & 1516.07 & $1.06 \times 10^{-5}$ \\
\hline & $3-21 G$ & 243.80 & 1713.36 & 7679.36 & $1.65 \times 10^{-5}$ \\
\hline \multirow[t]{3}{*}{$\mathrm{C}_{22} \mathrm{H}_{14}$} & STO-3G & 21.01 & 61.83 & 141.92 & $4.52 \times 10^{-6}$ \\
\hline & $3-21 G$ & 146.44 & 450.39 & 1132.11 & $5.07 \times 10^{-6}$ \\
\hline & $6-31 G^{*}$ & 636.19 & 2322.54 & 6744.89 & $1.76 \times 10^{-5}$ \\
\hline \multirow[t]{3}{*}{$\mathrm{C}_{30} \mathrm{H}_{18}$} & STO-3G & 38.97 & 160.54 & 511.93 & $1.09 \times 10^{-5}$ \\
\hline & $3-21 \mathrm{G}$ & 276.32 & 1121.20 & 3871.26 & $5.22 \times 10^{-6}$ \\
\hline & $6-31 G^{*}$ & 1252.76 & 6157.60 & 23433.20 & $2.23 \times 10^{-5}$ \\
\hline \multirow[t]{2}{*}{$\mathrm{C}_{42} \mathrm{H}_{24}$} & STO-3G & 74.23 & 333.83 & 1313.04 & $5.28 \times 10^{-6}$ \\
\hline & $3-21 \mathrm{G}$ & 533.89 & 2481.06 & 11476.33 & $5.66 \times 10^{-6}$ \\
\hline
\end{tabular}

The reported error is the maximum deviation over all matrix elements for a threshold of $10^{-7}$ with respect to the conventional calculation for a threshold of $10^{-11}$. 
ployed for calculations on selected linear alkanes, water clusters and small-gap linear polyacenes (i.e. from the series: benzene, napthalene, anthracene, ...) with thresholds of $10^{-7}$ and $10^{-11}$. Several factors can be assessed from this table: the role of basis set effects, the effect of different thresholds, and the effect of a smaller gap (for the polyacenes). Interestingly, larger reductions in computer time are observed when larger basis sets are employed. For example, computer time for evaluating all $K^{x}$ is reduced by a factor of 13.8 with the valence double zeta $3-21 \mathrm{G}$ basis set and 19.5 with the valence double zeta plus polarization $6-31 \mathrm{G}^{*}$ basis set for $\mathrm{C}_{24} \mathrm{H}_{50}$, using a threshold of $10^{-7}$. The corresponding factors are 14.6 and 21.9 for $\left(\mathrm{H}_{2} \mathrm{O}\right)_{20}$ and 7.7 and 10.6 for the $\mathrm{C}_{22} \mathrm{H}_{14}$ linear polyacene. The reason is simply because with larger basis sets many more ERIs are associated with small density matrix values, and therefore the thresholding strategy prunes more integrals and eliminates larger numbers of $x$ values from the contraction with the retained ERIs.

The value of the new method for small-gap systems can be assessed from the results in Table 1 for the linear polyacenes. Clearly it is not scaling linearly, but rather between $\mathrm{C}_{30} \mathrm{H}_{18}$ and $\mathrm{C}_{42} \mathrm{H}_{24}$ the scaling is approximately quadratic. However this is still more than enough to yield large savings relative to thresholding only the integrals in a cubic-scaling algorithm, as can be seen from the computational savings. By contrast, very little in the way of computational advantage would be expected for the LinK method applied to the ground state density matrix for these systems relative to conventional direct SCF. Similar considerations apply to the relatively small water clusters shown in Table 1: clearly LinK for multiple matrices becomes computationally valuable for much smaller systems than are needed to reach full linear scaling.

Table 1 also contains a measure of the extent to which numerical errors are present in the calculations. The error value reported is the maximum deviation of all calculated exchange matrix elements compared to the conventional calculation with only ERI thresholding. We see errors in exchange matrix elements that are larger than the specified contraction threshold for discarding in- dividual terms. This arises because many such individual terms contribute to a single exchange matrix element. Thus in practice the contraction threshold must be set roughly two or three powers of 10 smaller (tighter) than the target error in the exchange matrices themselves. There is no evidence of any significant growth in this error with molecular size, which is consistent with the fact that the errors must be due to local contributions.

\section{Conclusions}

A generalization of the LinK algorithm is presented for the problem of evaluating multiple local exchange matrices. This new method significantly reduces the computational cost while retaining controlled numerical precision. In contrast to LinK for a single matrix, significant computational savings are observed even for relatively small molecules by contracting multiple matrices. The large savings demonstrated here make it important to attack the corresponding problem of efficient evaluation of multiple Coulomb matrices. We hope to report on this shortly, as well as other improvements aimed at efficient analytical SCF force constant evaluation.

\section{Acknowledgements}

W.Z.L would like to express her deep gratitude to Prof. Arup K. Chakraborty and Mr. Baron Peters for many stimulating discussions relevant to this work. She also thanks the people who work in M.H.G.'s group for discussions and help in using the Q-CHEM package. Financial support from British Petroleum company as well as partial support (M.H.G) from the National Science Foundation (CHE-9981997) are gratefully acknowledged. C.O. acknowledges financial support by a Liebig 'Habilitation' fellowship from the FCI ('Fonds der Chemischen Industrie') and by an Emmy Noether research grant of the DFG ('Deutsche Forschungsgemeinschaft'). M.H.G. is on appointment as a Miller Research Professor in the Miller Institute for Basic Research in Science. 


\section{References}

[1] B.G. Johnson, C.A. White, Q. Zhang, B. Chen, R.L. Graham, P.M.W. Gill, M. Head-Gordon, in: J.M. Seminario (Ed.), Recent Developments in Density Functional Theory, vol. 4, Elsevier, Amsterdam, 1996, p. 441.

[2] R.E. Stratmann, G.E. Scuseria, M.J. Frisch, Chem. Phys. Lett. 257 (1996) 213.

[3] J.M. Perez-Jorda, W. Yang, Chem. Phys. Lett. 241 (1995) 469.

[4] L. Greengard, V. Rokhlin, J. Comput. Phys. 73 (1987) 325.

[5] L. Greengard, The Rapid Evaluation of Potential Fields in Particle Systems, MIT Press, Cambridge, MA, 1988.

[6] L. Greengard, Science 265 (1994) 909.

[7] C.A. White, B.G. Johnson, P.M.W. Gill, M. HeadGordon, Chem. Phys. Lett. 230 (1994) 8.

[8] C.A. White, B.G. Johnson, P.M.W. Gill, M. HeadGordon, Chem. Phys. Lett. 253 (1996) 268.

[9] C.A. White, M. Head-Gordon, J. Chem. Phys. 105 (1996) 5061.

[10] M.C. Strain, G.E. Scuseria, M.J. Frisch, Science 271 (1996) 51.

[11] E. Schwegler, M. Challacombe, J. Chem. Phys. 105 (1996) 2726.

[12] J.C. Burant, G.E. Scuseria, M.J. Frisch, J. Chem. Phys. 105 (1996) 8969.

[13] E. Schwegler, M. Challacombe, M. Head-Gordon, J. Chem. Phys. 106 (1997) 9708.

[14] E. Schwegler, M. Challacombe, Theoret. Chem. Accounts 104 (2000) 344.

[15] E. Schwegler, M. Challacombe, J. Chem. Phys. 111 (1999) 6223.
[16] C. Ochsenfeld, C.A. White, M. Head-Gordon, J. Chem. Phys. 109 (1998) 1663.

[17] C. Ochsenfeld, Chem. Phys. Lett. 327 (2000) 216.

[18] S. Yokojima, G.H. Chen, Chem. Phys. Lett. 292 (1998) 379.

[19] J. Almlöf, K. Faegri Jr., K. Korsell, J. Comput. Chem. 3 (1982) 385.

[20] J.A. Pople, R. Krishnan, H.B. Schlegel, J.S. Binkley, Int. J. Quantum Chem. Symp. S13 (1979) 225.

[21] M.J. Frisch, M. Head-Gordon, J.A. Pople, Chem. Phys. 141 (1990) 189.

[22] N.C. Handy, D.J. Tozer, G.J. Laming, C.W. Murray, R.D. Amos, Isr. J. Chem. 33 (1993) 331.

[23] A. Komornicki, G. Fitzgerald, J. Chem. Phys. 98 (1993) 1398.

[24] C. Ochsenfeld, M. Head-Gordon, Chem. Phys. Lett. 270 (1997) 399.

[25] H. Larsen, T. Helgaker, J. Olsen, P. Jorgensen, J. Chem. Phys. 115 (2001) 10344.

[26] J.C. Burant, M.C. Strain, G.E. Scuseria, M.J. Frisch, Chem. Phys. Lett. 258 (1996) 45.

[27] R.E. Stratmann, J.C. Burant, G.E. Scuseria, M.J. Frisch, J. Chem. Phys. 106 (1997) 10175.

[28] J. Cullen, Chem. Phys. 202 (1996) 217.

[29] T. Van Voorhis, M. Head-Gordon, Chem. Phys. Lett. 317 (2000) 575.

[30] T. Van Voorhis, M. Head-Gordon, J. Chem. Phys. 115 (2001) 7814 .

[31] V. Dyczmons, Theoret. Chim. Acta 28 (1973) 307.

[32] R. Ahlrichs, Theoret. Chim. Acta 33 (1974) 157.

[33] M. Häser, R. Ahlrichs, J. Comput. Chem. 10 (1989) 104.

[34] M. Head-Gordon, J.A. Pople, J. Chem. Phys. 89 (1988) 5777.

[35] J. Kong et al., J. Comput. Chem. 21 (2000) 1532. 